\title{
European Association for the Study of Diabetic Eye Complications (EASDEC) 1991
}

\author{
J.S. K. Wong and E.M.Kohner \\ Royal Postgraduate Medical School, Hammersmith Hospital, London, UK
}

The inaugural meeting of EASDEC was held at Hammersmith Hospital in London on the 8-9 September 1991 and covered topics on cell biology, functional abnormalities in diabetic retinopathy and the problem of cataracts in diabetic patients.

Dr. M. Lorenzi (Boston, USA) gave a keynote lecture on the role of cell biology in understanding the pathogenesis of diabetic retinopathy. She cautioned against extrapolating the findings of in vitro studies to the in vivo situation as clearly there are difficulties in simulating the extracellular matrix (as well as the metabolic milieu) in which retinal capillary endothelial cells and pericytes are cultured. This is a particular problem in diabetes, as basement membrane abnormalities are early manifestations of the disease and endothelial cells cultured in high glucose have demonstrated increased synthesis of extracellular proteoglycan components such as fibronectin, laminin and type IV collagen. Despite these caveats, the cell biology approach continues to give an important insight into the pathogenesis of diabetic retinopathy and provides a rationale for conducting in vivo studies. Mr. Campos (Pennsylvania State, USA) reported on the characterization of histamine receptors in bovine retinal capillary endothelial cells and their putative role in blood retinal barrier permeability, while Dr. M. Boulton's group (Manchester, UK) demonstrated that replication of bovine retinal capillary endothelial cells were inhibited by culture in increasing oxygen tensions. Dr. Porta (London, UK) examined the effect of a number of growth factors on DNA synthesis in bovine retinal capillary cells, whilst Dr. Chibbers (London, UK) presented an interesting paper on the preferential glycation of pericyte cytoplasmic actin when these cells were cultured in high $(30 \mathrm{mmol} / \mathrm{l})$ glucose media.

Dr. H.Lund-Anderson (Hellerup, Denmark) reviewed the numerous functional abnormalities that may be encountered during the course of diabetic retinopathy and attempted to correlate abnormalities in blood retinal barrier (BRB) permeability with the anatomical sequelae of diabetic retinopathy. Prof. T.M.Hollis (Pennsylvania State, USA) reported in both animal and human studies that $\mathrm{H}_{1}$ antagonists (astemizole) appeared to reduce BRB permeability, while Dr. C.B.Engler (Hellerup, Denmark) in preliminary studies showed that angiotensin converting enzyme inhibition has no apparent effect on BRB in the short term. Dr. V.Patel (London, UK) presented data showing that retinal blood flow velocity as assessed with Laser Doppler Velocimetry increased with increasing severity of diabetic retinopathy but fell following pan-retinal photocoagulation for proliferative retinopathy. Dr. S. Rassam (London, UK) reported early findings of retinal blood flow responses to tyramine induced increases in systemic blood pressure in both normal volunteers and diabetic patients. Autoregulation appeared to be maintained until $40 \%$ rise in mean arterial pressure is achieved when the autoregulatory responses in diabetic patients appeared to be impaired. Using the technique of Scanning Laser Ophthalmoscopy, Dr. Wolf (Aachen, Germany) was able to elegantly demonstrate that the foveal avascular zone and perifoveal intercapillary areas in diabetic patients were significantly enlarged compared to normal control subjects. However, increasing duration of diabetes had no effect on mean capillary blood flow velocity.

Mr. E. Rosen (Manchester, UK) addressed the problem of cataract surgery in diabetic patients. He favoured the opinion that all diabetic patients should have intra-ocular lens implant as the aim was to improve visual acuity and the dioptre of the lens should be as large as possible. Where possible, heparinised lens should be used as less fibrinous deposits are encountered with this type of lens thus reducing the problem of photocoagulation due to inadequate fundal view. The incidence and type of post-operative complications was obviously increased in diabetic patients and this was discussed in subsequent papers. Mr. P.G. Hykin (London, UK) showed that diabetic patients with background retinopathy had a poorer visual outcome following cataract surgery compared to non-diabetic patients and the visual prognosis is worse in those with proliferative retinopathy with some eyes developing advanced diabetic retinopathy (rubeosis, retinitis proliferans and vitreous haemorrhage) following surgery. The incidence of exudative maculopathy was also increased post-operatively in eyes with background retinopathy. The problem of post-operative maculopathy following cataract extraction was confirmed by the findings of Dr. G. Chaine (Paris, France), and Dr. U.Menchini (Milan., Italy). Dr. J. Weik (London, UK) in an audit of cataract surgery in patients with diabetic retinopathy demonstrated that the pupillary diameter was decreased in more than half of the eyes post-operatively and pointed out that this posed problems with laser treatment in inadequately dilated pupils. The general consensus from all these papers is that where possible adequate photocoagulation should be carried out before cataract surgery to reduce post-operative complications and to avoid the problems of laser photocoagulation in patients with poorly dilated pupils.

Overall, this was an auspicious inauguration of EASDEC and the abstracts of this meeting will be published in the European Journal of Ophthalmology. Subsequent annual meetings are planned to immediately precede the main EASD conference. The venue for EASDEC 1992 has yet to be finalised but Vienna has been tentatively suggested as a likely successor to London. This will be advertised in due course in the Diabetic and Ophthalmology journals. Watch this space!

Acknowledgements. The EASDEC meeting was supported in part by NovoNordisk, Pfizer and Servier.

Prof. E. Kohner

Medical Ophthalmology

Hammersmith Hospital

Du Cane Road 